\title{
Anthracycline induced cardiotoxicity in adult cancer patients: a prospective cohort study from a specialized oncology treatment centre in Uganda
}

\author{
Solomon Kibudde ${ }^{1}$, Charles Kiiza Mondo ${ }^{2}$, Davis Kibirige ${ }^{3}$, Victoria Walusansa ${ }^{1}$, Jackson Orem ${ }^{1}$
}

1. Uganda Cancer Institute, Department of Medical Oncology.

2. Mulago Hospital, Department of Medicine, Division of Cardiology.

3. Uganda Martyrs Hospital Lubaga, Department of Medicine.

\begin{abstract}
:
Purpose: To determine the cumulative incidence of anthracycline induced cardiotoxicity (AIC), its predictors, and associated electrocardiographic and echocardiographic manifestations in adult cancer patients at Uganda Cancer Institute (UCI).

Methods: We enrolled 160 participants between June 2013 and April 2014 and followed them up for a median of 146 days. Data on clinical, electrocardiographic and echocardiographic findings was obtained at baseline, and at completion of chemotherapy. The Pearson chi square test was used to identify the predictors associated with cardiotoxicity.

Results: Of the 64 patients who were accessible for follow-up electrocardiography (ECG) and echocardiography (ECHO), fourteen participants developed cardiotoxicity hence a cumulative incidence rate of $21.9 \%$ with 95\% CI 13.5\%- 33.43\%. The predictors of AIC were female gender $(\mathrm{p}=0.025), \operatorname{LVEF}(\mathrm{p}=0.014)$ and LVFS $(\mathrm{P}=0.019)$. Anthracycline therapy was associated with shortening of the QRS duration $(84.3 \pm 7.9 \mathrm{Vs} 82.1 \pm 11.8 \mathrm{~ms}, \mathrm{p}=0.005)$, prolongation of the QTc interval (411.9 $\pm 30.7 \mathrm{Vs}$ $447.2 \pm 39.4 \mathrm{~ms}, \mathrm{p}=<0.001)$ and reduction in the LVEF (66.4 $\pm 7.7 \mathrm{Vs} 63.9 \pm 8.4 \%, \mathrm{p}=0.026)$ and LVFS $(36.9 \pm 6.2 \mathrm{Vs} 35.1 \pm 6.6 \%$, $\mathrm{p}=0.03)$.
\end{abstract}

Conclusion: The cumulative incidence of AIC in this study cohort was high. Our findings emphasize the need for early monitoring for AIC.

Keywords: Anthracycline induced cardiotoxicity, cancer patients, Uganda.

DOI: https://dx.doi.org/10.4314/ahs.v19i1.40

Cite as: Kibudde S, Mondo CK, Kibirige D, Walusansa V, J O. Anthracycline induced cardiotoxicity in adult cancer patients: a prospective cohort study from a specialized oncology treatment centre in Uganda. Afri Health Sci. 2019;19(1). 1647-1656. bttps://dx.doi.org/10.4314/ahs. v19i1.40

\section{Introduction}

Anthracyclines have been associated with irreversible cardiotoxicity and progression to cardiomyopathy and heart failure $^{1}$. These highly potent anti-neoplastic agents are used to treat cancers of the breast, stomach, liver, esophagus, as well as sarcomas, leukemia, lymphomas and myeloma.

\section{Corresponding author: \\ Solomon Kibudde, \\ Uganda Cancer Institute, \\ Department of Medical Oncology. \\ Email: skibudde@yahoo.com}

Cardiotoxicity is defined as one or more of the following: cardiomyopathy in terms of reduction in the left ventricular ejection fraction (LVEF), either global or more severe in the septum; symptoms associated with heart failure $(\mathrm{HF})$, signs associated with heart failure such as S3 gallop, tachycardia or both, reduction in LVEF from baseline that is in the range of less than or equal to $5 \%$ to less than $55 \%$ with accompanying signs or symptoms of heart failure, or a reduction in LVEF in the range of equal to or greater than $10 \%$ to less than $55 \%$, without accompanying signs or symptoms of $\mathrm{HF}^{2}$.

Anthracycline induced cardiotoxicity is commonly categorized according to the time of onset as sub-acute, acute or chronic. Sub-acute and acute cardiotoxicity occur between the time of chemotherapy initiation to up 
to 2 weeks after completion of treatment while chronic cardiotoxicity is further classified into early cardiotoxicity (onset within one year of chemotherapy initiation) and late (onset after one year of chemotherapy initiation) $)^{3,4}$. Sub acute and acute cardiotoxicity which occurs in up to $20-30 \%$ patients comprises of transient electrophysiological abnormalities. These include non-specific ST- and T-wave flattening, decreased QRS voltage and prolongation of QTc interval, sinus tachycardia and supra-ventricular tachycardia. Chronic cardiotoxicity is often associated with an irreversible non ischemic dilated cardiomyopathy and intractable heart failure ${ }^{5}$.

The plausible theory to explain how anthracyclines induce cardiac damage is by free oxygen rich radicals generated by the anthracycline-iron complexes. This leads to progressive left ventricular dilatation, left ventricular wall thinning and decreased contractility (irreversible dilated cardiomyopathy) ${ }^{6}$.

In sub-Saharan Africa (SSA), there is a noted paucity of information on burden of anthracycline induced cardiotoxicity and related predictors among adult cancer patients receiving anthracycline chemotherapy. Most oncology centers lack well established guidelines on cardiac monitoring of patients on cancer chemotherapy, with majority of patients receiving cardiac evaluation only prior to initiation of chemotherapy.

We therefore conducted this prospective cohort study to describe the incidence and predictors of anthracycline induced cardiotoxicity among adult cancer patients receiving an anthracycline containing treatment regimen at the Uganda Cancer Institute (UCI), a specialized oncology treatment centre in Kampala, Uganda.

\section{Methods}

\section{Study design and participants}

We conducted a prospective cohort study to describe the incidence and predictors of anthracycline induced cardiotoxicity among adult patients receiving anthracycline based chemotherapy at the Uganda Cancer Institute (UCI) between the period June 2013 and April 2014. Patients aged 18 years and older, with histologically confirmed diagnosis of cancer and a doctor's prescription with an anthracycline-containing regimen were consecutively sampled. We excluded patients with history of prior treatment with any anthracycline chemotherapy drugs or herceptin/transtuzumab.

One hundred sixty (160) participants met the inclusion criteria and were followed up for up to three months after completion of chemotherapy. Only 64 participants were available for the repeat evaluation with ECG and ECHO at three months after completion of chemotherapy as shown in Figure 1 below.

\section{Sample size estimation \\ Study procedures Treatment, patient evaluation and study procedures}

All patients were treated according to the standard of care at the Uganda Cancer Institute, and the treatment regimens are summarized in table 2 below. Pre-tested and pre coded questionnaires were used to collect the study information in three categories namely; the patients factors (age, gender, education, presence of cardiac symptoms, family history of cardiovascular disease, hypertension, BMI, diabetes, performance status and tobacco smoking); cancer related factors (cancer type, stage and prior chest irradiation); and chemotherapy related factors (cumulative dose, type of anthracycline and combination therapy with cyclophosphamide).

All the study cardiac evaluations with ECG and ECHO were done at the cardiac laboratory, Mulago National Referral and Teaching Hospital, Uganda; by a certified cardiologist. A 12 lead resting ECG was done using the Phillips Page Writer Trim III Model. The different readings of the heart rate (HR), QRS axis, PR interval, QRS duration and QTc interval as given by the machine were documented and interpretation of the ECG was performed by the study cardiologist.

The echocardiographic evaluation was done using the Phillips HDX10 version employing several modalities including the two-dimensional echocardiography, M-mode and Doppler echocardiography. All the ECHO dimensions were obtained as per the American Society of Echocardiography recommendations ${ }^{9}$.

\section{Outcome measures}

A repeat ECG and ECHO were done after completion of chemotherapy and all the data was reviewed by the cardiologist. The primary end-point was a reduction in 
the left ventricular ejection fraction (LVEF) of at least 10 percent with or without the presence of symptoms of heart failure. The difference in the LVEF was computed manually by comparing the pre-treatment LVEF to the post-treatment LVEF. Other secondary measurements were the change in the left ventricle fractional shortening (LVFS), the PR interval, QRS duration and the QTc interval.

\section{Data management statistical methods}

Data was entered using EPI-DATA (version 3.1) software and exported to STATA 12.0, for analysis. Continuous variables (age, weight, Height, BMI, hemodynamic parameters, cumulative anthracycline dose, electrocardiographic and echocardiographic evaluation parameters) were all presented in the form of means, standard deviations (SD), medians and inter quartile ranges (IQR).

Categorical variables (gender, type of cancer, type of anthracycline, outcome variables) were presented in frequencies and percentages. The Pearson chi square test was used to identify the predictors associated with cardiotoxicity. Variables with a p value $\leq 0.05$ were considered as statistically significant.

\section{Ethical considerations}

Ethical approval was obtained from the Department of Medicine, Makerere University College of Health Sciences, the School of Medicine Research and Ethics Committee (SOMREC) and the Ethics and Research Committee of Uganda Cancer Institute. In addition, signed written informed consent was obtained from every participant using board approved consent forms in either English or a translated local language.

\section{Results \\ Socio demographic and clinical characteristics of study participants}

Of the 160 study participants enrolled, majority were females $(124,77.50 \%)$ giving a female to male ratio of 3.4 : 1. The mean age of the study participants was 42.29 years (SD -13.73 years). Cancer of the breast was the most predominant diagnosis in this study cohort $(n=110,68.75 \%)$ followed by Non-Hodgkin's Lymphoma ( $\mathrm{n}=21 ; 13.13 \%$ ). All the 160 patients had advanced stages of disease (stage III/IV) and the majority had a good performance status between level 0 and 1 ( $n=147,92.5 \%)$. The performance status was assessed using the scale developed by the Eastern Cooperative Oncology Group (ECOG). Nine (9) patients were excluded because they did not receive any chemotherapy due to disease progression and early loss to follow up. Sixteen patients $(10 \%)$ died during the period of follow up, while 80 patients $(50 \%)$ patients were lost to follow up before completion of chemotherapy, and only 64 patients returned for follow up cardiac evaluation within three months of chemotherapy completion (Figure 1 summarizes the patient flow during the study). Of the 64 patients who had follow up cardiac evaluation within three months after completion of chemotherapy, $48(80 \%)$ were female. Their mean age was $44.3+13.1$ years and the majority $(43,67.2 \%)$ had breast cancer. The mean values for the full hemogram, liver and renal function tests for all the patients in this study cohort were all within normal limits. The socio-demographic and clinical profile of the study participants is shown in Table 1. 


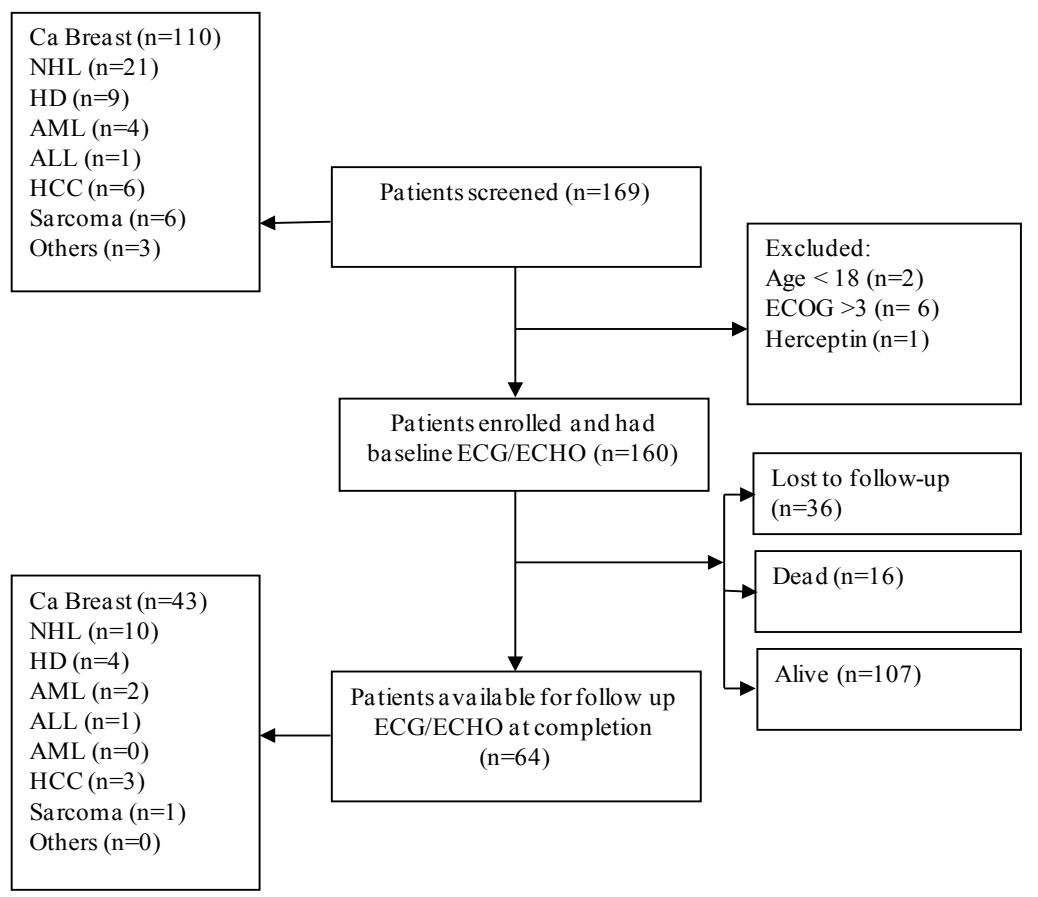

Figure 1: Study flow diagram showing the cohort at different stages

Note: NHL - Non-Hodgkin's Lymphoma, HD - Hodgkin's Disease, AML - Acute Myeloid Leukemia, ALL - Acute Lymphoblastic Leukemia, HCC - Hepatocellular Carcinoma, ECG - Electrocardiogram, ECHO-Echocardiogram, ECOG - Eastern Cooperative Oncology Group

Table 1: Socio- demographic and clinical characteristics of the study participants

\begin{tabular}{lr}
\hline Characteristic & Cases $(\mathrm{N}=160)$ \\
\hline Age, median (IQR) & $45.3(35.3-55.8)$ \\
Gender & \\
$\quad$ Female, n (\%) & $124(77.5 \%)$ \\
BMI, median (IQR) & $24.6(21.2-28.1)$ \\
\hline HIV sero positive, n (\%) & $27(16.9 \%)$ \\
ECOG, n (\%) & $147(92.5 \%)$ \\
$\quad$ 0/1 & $12(7.5 \%)$ \\
$\quad$ //3 & \\
Stage, n (\%) & $0(0 \%)$ \\
$\quad$ I/II & $86(64.2 \%)$ \\
$\quad$ III & $48(35.8 \%)$ \\
$\quad$ IV & $12.3(10.6-14.1)$ \\
Hb, median (IQR) & $16.7(12.0-23.1)$ \\
ALT, median (IQR) & $23.7(20.2-33.1)$ \\
\hline AST, median (IQR) & $75.0(61.3-83.8)$ \\
Cr, median (IQR) & $270(197-330)$ \\
\hline LDH, median (IQR) & \\
\hline
\end{tabular}

Note: BMI - Body mass Index, $\mathrm{Hb}$ - Hemoglobin, WCC - White Cell Count, Plt - Platelet, ALT - Alanine Transaminase, AST - Aspartate Transaminase, T-Prot - Total Protein, Cr - Creatinine, $\mathrm{LDH}$ - Lactate Dehydrogenase. 


\section{Chemotherapy regimens used during the study}

The cancer chemotherapy regimens used for patients enrolled in this cohort are shown in Table 2. Doxorubicin alone or in combination with cyclophosphamide was contained in chemotherapy prescriptions of majority of patients ( $\mathrm{n}=155,96.88 \%)$. The most prescribed treatment dose of Doxorubicin was $100 \mathrm{mg} / \mathrm{m}^{2}$ especially in the chemotherapy regimen for breast cancer.

\section{Table 2: Diagnosis and schedule of chemotherapy among the patients evaluated for anthracycline-induced cardiotoxicity}

\begin{tabular}{lllll}
\hline Diagnosis & Patients. (n=160) & Regimen & Schedule & Cycles received \\
\hline Breast cancer & $110(68.8 \%)$ & $\mathrm{CA}^{\dagger} \mathrm{F}$ & 3 weekly & $6(4-6)$ \\
NHL & $21(13.1 \%)$ & CHOP & 3 weekly & $3(2-6)$ \\
HD & $09(5.6 \%)$ & $\mathbf{A}^{\dagger} \mathrm{BVD}$ & 2 weekly & $3(2-6)$ \\
AML & $04(2.5 \%)$ & Dauno/AraC & Daily & $3(3-5)$ \\
ALL & $01(0.6 \%)$ & $\mathrm{DVPA}^{\ddagger}$ & Daily & $3(3-3)$ \\
HCC & $06(3.7 \%)$ & Doxorubicin & Weekly & $6(3-6)$ \\
Sarcomas & $06(3.7 \%)$ & $\mathbf{A}^{\dagger} \mathrm{D}$ & 3 weekly & $6(0-6)$ \\
\hline
\end{tabular}

Note: $\mathrm{A}^{\dagger}$ - Adriamycin/(Doxorubicin/Hydroxydaunorubicin[H]), $\mathrm{A}^{\ddagger}-\mathrm{L}$-Asparaginase, AraC - Cytarabine, B -Bleomycin, C - Cyclophosphamide, D - Dacarbazine, F - \%-Fluorouracil, O - Oncovin/(Vincristine[V]), $\mathrm{P}$ - Prednisone, $\mathrm{H}-/($ Adriamycin/Doxorubicin)

\section{Cumulative incidence of anthracycline induced car- diotoxicity}

The median duration of follow up of the study participants was 146 days with a range of 23 days to 260 days. Fourteen (14) out of 64 patients developed anthracycline induced cardiotoxicity within three months of completing anthracycline based chemotherapy; hence the cumulative incidence rate of $21.9 \%$ with $95 \%$ confidence intervals $(13.50 \%-33.43 \%)$. Only one person developed overt signs and symptoms of heart failure.
Predictors of anthracycline induced cardiotoxicity; Pearson chi square test was used to identify the statistically significant variables associated with anthracycline induced cardiotoxicity; only female gender $(p=0.025)$, pre-treatment LVEF $(\mathrm{p}=0.014)$ and pre-treatment LVFS (0.019) were noted

Clinical, electrocardiographic and electrocardiographic characteristics associated with anthracycline induced cardiotoxicity were female gender $(p=0.025)$, pre-treatment LVEF $\leq 55 \%(p=0.001)$ and pre-treatment LVFS $\leq 30 \%$ $(p=0.04)$ (illustrated in table 3). 
Table 3: Clinical, electrocardiographic and electrocardiographic characteristics associated with anthracycline induced cardiotoxicity

\begin{tabular}{|c|c|c|c|c|c|}
\hline \multirow[t]{2}{*}{ Variable } & & \multicolumn{2}{|c|}{$\begin{array}{l}\text { Anthracycline induced } \\
\text { cardiotoxicity }\end{array}$} & \multirow{2}{*}{$\begin{array}{l}\text { Chi } \\
\text { square } \\
\left(\mathrm{X}^{2}\right)\end{array}$} & \multirow[t]{2}{*}{$\mathrm{p}$-value } \\
\hline & & $\begin{array}{l}\text { Cardiotoxicity } \\
(\mathrm{N}=14)\end{array}$ & $\begin{array}{l}\text { No Cardiotoxicity } \\
(\mathrm{N}=50)\end{array}$ & & \\
\hline \multirow[t]{2}{*}{ Gender } & Male & $0(0.00 \%)$ & $14(28.00 \%)$ & 5.02 & 0.025 \\
\hline & Female & $14(100.00 \%)$ & $36(74.00 \%)$ & & \\
\hline \multirow[t]{2}{*}{ Age (years) } & $18-49$ & $10(71.4)$ & $33(66.0)$ & 0.15 & 0.702 \\
\hline & $50-73$ & $4(28.6)$ & $17(34.0)$ & & \\
\hline \multirow[t]{2}{*}{ Education $^{+}$} & Post-primary & $5(35.7)$ & $24(51.1)$ & 1.02 & 0.97 \\
\hline & Yes & $2(14.29 \%)$ & $9(18.00 \%)$ & & \\
\hline \multirow[t]{2}{*}{ Tobacco smoking } & No & $14(100.00 \%)$ & $48(96.00 \%)$ & 0.58 & 0.447 \\
\hline & Yes & 0 & $2(4.00 \%)$ & & \\
\hline \multirow[t]{2}{*}{ HIV } & No & $13(92.86 \%)$ & $38(76.00 \%)$ & 1.92 & 0.166 \\
\hline & Yes & $1(7.14 \%)$ & $12(24.00 \%)$ & & \\
\hline \multirow[t]{2}{*}{ Diabetes } & No & $14(100.00 \%)$ & $48(96.00 \%)$ & 0.58 & 0.447 \\
\hline & Yes & 0 & $2(4.00 \%)$ & & \\
\hline \multirow[t]{3}{*}{ ECOG } & 0 & $5(35.71 \%)$ & $15(30.00 \%)$ & 0.48 & 0.787 \\
\hline & 1 & $8(57.14 \%)$ & $33(66.00 \%)$ & & \\
\hline & 2 & $1(7.14)$ & $2(4.00 \%)$ & & \\
\hline \multirow[t]{3}{*}{ LVEF } & $<55$ & $6(60.0 \%)$ & $4(40.00 \%)$ & 18.7 & $<0.001$ \\
\hline & $55-65$ & $8(33.3 \%)$ & $16(66.7 \%)$ & & \\
\hline & $>65$ & $0(0.00 \%)$ & $30(100 \%)$ & & \\
\hline \multirow[t]{3}{*}{ LVFS } & $<30$ & $6(46.1 \%)$ & $7(53.9 \%)$ & 6.43 & 0.040 \\
\hline & $30-45$ & $8(14.29 \%)$ & $38(82.6 \%)$ & & \\
\hline & $>45$ & $0(0.00 \%)$ & $5(100.0 \%)$ & & \\
\hline \multirow[t]{2}{*}{$\mathrm{E}: \mathrm{A}$} & $0.6-1$ & $8(22.9 \%)$ & $27(77.1 \%)$ & 0.02 & 0.892 \\
\hline & $>1$ & $6(21.4 \%)$ & $22(78.6 \%)$ & & \\
\hline \multirow[t]{2}{*}{ Cumulative dose } & $<550 \mathrm{mg} / \mathrm{m} 2$ & $9(21.4)$ & $33(78.6)$ & 0.28 & 0.594 \\
\hline & $>550 \mathrm{mg} / \mathrm{m} 2$ & $5(27.8)$ & $13(72.2)$ & & \\
\hline
\end{tabular}

Statistically significant $P<0.05$

However, we observed these significant differences in ECG and ECHO parameters at 3 months after anthracycline treatment: shortening of the QRS duration (84.3 $\pm 7.9 \mathrm{~ms}$ Vs $82.1 \pm 11.8 \mathrm{~ms}, \mathrm{p}=0.005)$, prolongation of the QTc interval $(411.9 \pm 30.7 \mathrm{~ms}$ Vs $447.2 \pm 39.4 \mathrm{~ms}$, $\mathrm{p}=<0.001)$ and reduction in the LVEF $(66.4 \pm 7.7 \%$ Vs $63.9 \pm 8.4 \%, p=0.026)$ and LVFS $(36.9 \pm 6.2 \% \mathrm{Vs}$ $35.1 \pm 6.6 \%, \mathrm{p}=0.03$ ) (illustrated in table 4 below). 
Table 4: Difference in the electrocardiographic and echocardiographic parameters withinthree months after completion of chemotherapy

\begin{tabular}{|c|c|c|c|}
\hline & Baseline & End of Chemotherapy & P-value \\
\hline \multicolumn{4}{|l|}{ ECG parameters } \\
\hline PR interval (mSec) & $154.2 \pm 27$ & $154.2 \pm 24.1$ & 0.979 \\
\hline QRSduration (mSec) & $84.27 \pm 7.9$ & $82.1 \pm 11.8$ & 0.005 \\
\hline QTe interval (mSec) & $411.9 \pm 30.7$ & $447.2 \pm 39.4$ & $<0.001$ \\
\hline \multicolumn{4}{|l|}{ ECHO parameters } \\
\hline LVEF (\%) & $66.4 \pm 7.7$ & $63.9 \pm 8.4$ & 0.026 \\
\hline LVFS (\%) & $36.9 \pm 6.2$ & $35.1 \pm 6.6$ & 0.030 \\
\hline E:A Ratio & $1.07 \pm 0.3$ & $1.03 \pm 0.3$ & 0.278 \\
\hline
\end{tabular}

With regard to the measured LVEF, we observed a greater reduction in the LVEF among the age group of 18 -45 years as compared to other age groups (illustrated in figure 2).
This observation could be explained by the relatively higher number of patients in the age group 18-45 years who had a follow up measure of the LVEF after completion of chemotherapy. The change in the LVEF was higher among patients with hematological malignancies compared to those with solid tumors (illustrated in figure 3).

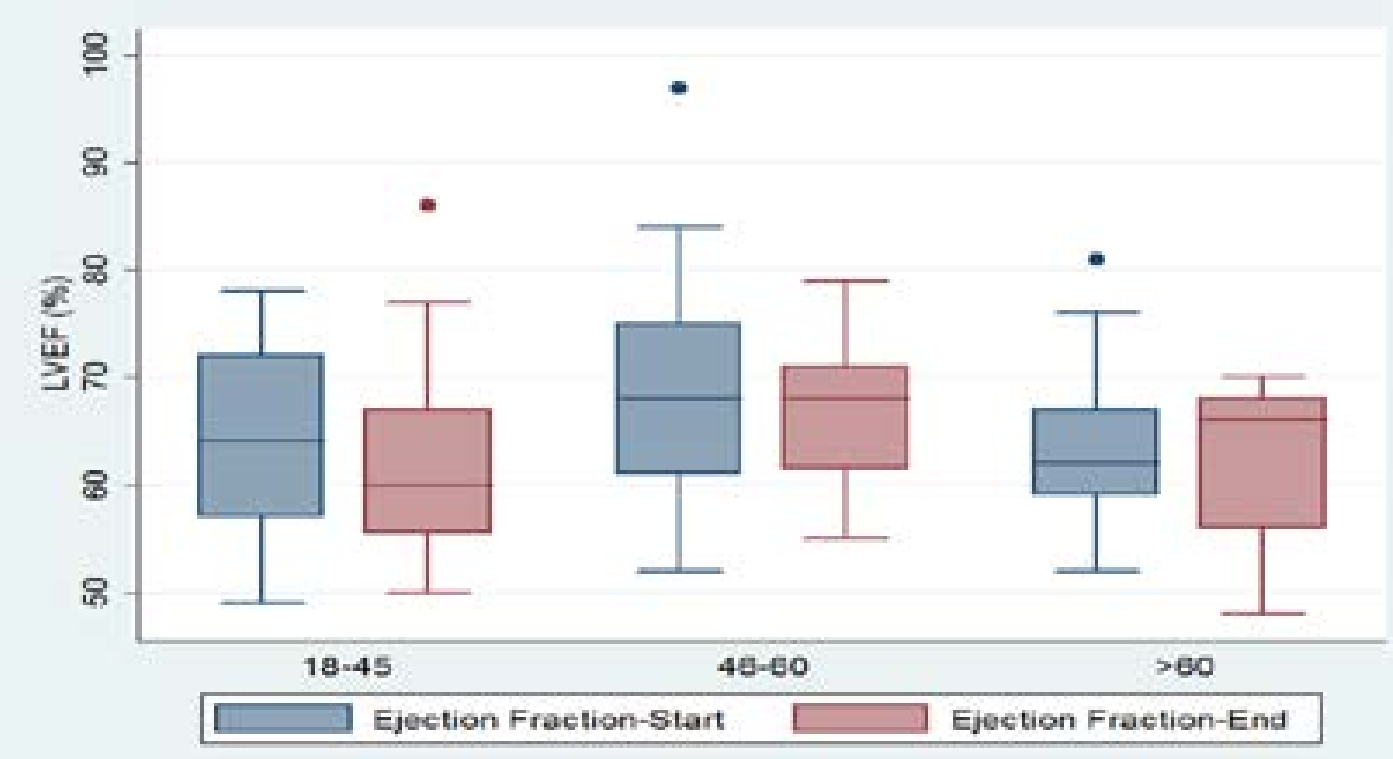

Figure 2 showing the change in ejection fraction by the different age groups. 


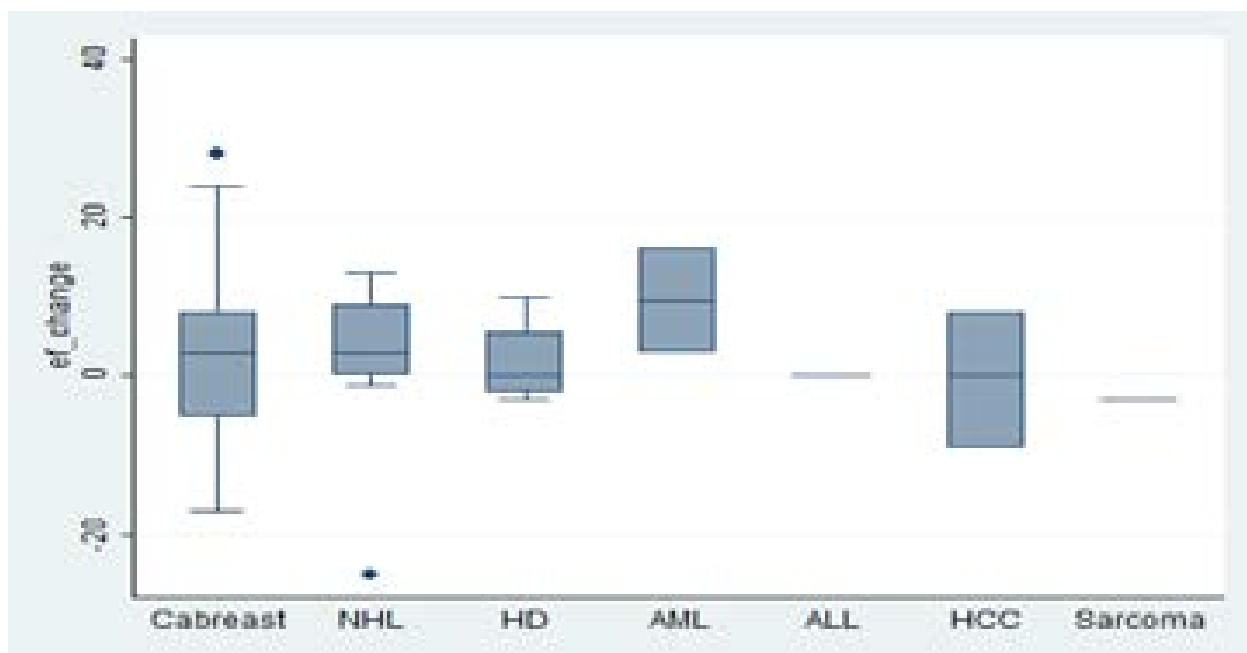

\section{Figure 3: Box plot showing change in left ventricle ejection fraction according to the different cancers.}

\section{Discussion}

To our knowledge, this is the first prospective cohort study to examine the incidence and predictors of anthracycline induced cardiotoxicity in adult cancer patients in Uganda. Our findings demonstrate a high incidence of anthracycline-induced cardiotoxicity in this cohort with one among every five patients $(21.9 \%)$ developing significant reduction in the left ventricular ejection fraction. Furthermore, the predictors for anthracycline induced cardiotoxicity were female gender, and a reduction in the left ventricular ejection fraction and/or the left ventricle fraction shortening. The significant ECG changes were shortening of the QRS duration, and prolongation of the QTc interval.

Anthracycline-induced cardiotoxicity is a dose-dependent toxicity with a recommended threshold of $550 \mathrm{mg} / \mathrm{m}^{2}$ for Adriamycin (doxorubicin); in our cohort, nearly one third of patients (18 out of the 64 participants) exceeded the ceiling dose of anthracycline chemotherapy. Secondly, the presentation is sub-clinical in majority of patients; and hence the importance of clinical vigilance among patients receiving anthracycline-based chemotherapy. Reduction in left ventricle ejection fraction was consistent with increased risk for cardiac dysfunction as previously reported. ECG findings associated with anthracycline-induced cardiotoxicity remain non-specific but could be predictive. The shortening of the QRS duration implies a reduction of the myocardium depolarization time hence increasing the myocardium repolarization time.

Our findings are in-line with similar studies including three from Africa with reported incidence of anthracycline induced cardiotoxicity ranging from $0 \%$ to $47 \%{ }^{10-14}$. In Africa, two studies done among adult cancer patients in Cote $\mathrm{d}$ ívore and Morocco show a high incidence of anthracycline-induced cardiotoxicity. Elalouani $\mathrm{C}$ et al who conducted a prospective cohort study in Morocco investigating the frequency of anthracycline induced cardiotoxicity noted that $56 \%$ of the 70 patients developed a decrease in cardiac function and $4 \%$ of cases developed severe cardiotoxicity ${ }^{15}$. In another prospective cohort study by Pio $\mathrm{M}$ et al conducted at Abidjan institute of cardiology, Cote d ívore over 10 months, 45 adult patients were followed up and 4 (8.8\%) developed significant cardiotoxicity ${ }^{16}$.

In another study by Shiroya -Wandabwa $\mathrm{M}$ et al done in 110 children being treated with doxorubicin with a cumulative dose $>200 \mathrm{mg} / \mathrm{m}^{2}$ at Kenyatta National Hospital, Nairobi, Kenya, a comparable point prevalence of anthracycline induced cardiotoxicity of $29 \%, 95 \%$ CI $21.2-$ 37.9 was documented ${ }^{17}$.

\section{Predictors of anthracycline induced cardiotoxicity}

Described predictors of anthracycline induced cardiotoxicity include: the type of anthracycline, cumulative dose, and presence of additional risk factors for developing 
heart damage such as chest irradiation, tumor type, combination chemotherapy with cyclophosphamide, female gender, extremes of ages and malnutrition ${ }^{5,18}$. In our study, we identified female gender, LVEF and LVFS as strongly predictive of anthracycline induced cardiotoxicity. Of note, the majority of our patients were female, and all patients received combination chemotherapy with mostly cyclophosphamide.

\section{Clinical, electrocardiographic and echocardiograph-} ic features of anthracycline induced cardiotoxicity

In this study, the following variables were noted to be associated with anthracycline induced cardiotoxicity: female gender, $\mathrm{LVEF} \leq 55 \%$ and LVFS $\leq 30 \%$. The significant ECG and ECHO changes that were documented 3 months after completion of chemotherapy included: shortening of the QRS duration, prolongation of the QTc interval, and reduction in the LVEF (illustrated in table 3). These observations are in agreement with findings from previous studies and in literature ${ }^{14,19-21}$.

The co-morbid conditions and clinical features that we evaluated included presence of hypertension, diabetes mellitus, history of angina or heart failure or sudden death, family history of any cardiovascular disease, cardiac symptoms (dyspnoea on exertion, chest discomfort, ankle swelling, orthopnoea and easy fatigability) and they were all not associated with cardiotoxicity. This probably could have been due to limited number of patients evaluated in this study.

\section{Study limitations}

We encountered several limitations during the conduct of this research. First, there was a high rate of loss to follow up attributed to fluctuation in the availability of chemotherapy at UCI; compounded by low education level, family's myths about cancer treatment and financial constraints especially transport fares. Secondly, we recognize that the study follow-up period was short thus limiting the events of cardiotoxicity that could be observed. Thirdly, during this study, we were unable to perform serial echocardiography prior every cycle of chemotherapy so as to determine the exact time when the cardiotoxicity sets in; and lastly, we were unable to do Tissue Doppler Imaging (TDI) to assess diastolic function; this makes it difficult to differentiate true versus pseudo diastolic heart dysfunction just based on the E: A ratios we observed.

\section{Conclusion}

Our findings demonstrate a high incidence of anthracycline induced cardiotoxicity within three months of completion of chemotherapy in this study cohort. Therefore, we strongly recommend that all cancer patients receiving anthracycline based chemotherapy should have cardiac evaluation with at least a left ventricle ejection fraction (LVEF) before, during and after completion of chemotherapy.

\section{Acknowledgements}

Many thanks to the study team particularly Agnes Wateera, Robert Buyungo, Susan Munyonyo (RIP), Doreen Katushabe, the entire MEPI CVD-Linked program team (Prof Nelson K Sewankambo, Prof Moses R Kamya, Dr Chris Longerneck, Dr Isaac Sssinabulya and Dr Yvone Nabunya) for the invaluable support towards this research.

This research was funded through support from Fogarty International Center, the National Heart Lung and Blood Institute, and the Common Fund of the National Institutes of Health under Award Number 5R24 TW008861. The content is solely the responsibility of the authors and does not necessarily represent the official views of the National Institutes of Health.

Lastly, we are extremely grateful to our patients who participated in this cohort for volunteering their precious time and efforts to comply to the study procedures.

\section{Conflict of interest}

None.

\section{References}

1. Elliott P. Pathogenesis of Cardiotoxicity Induced by Anthracyclines Seminars in oncology. 2006;33:2-7.

2. Seidman A, Hudis C, Pierri MK, Shak S, Paton V, Ashby M, et al. Cardiac dysfunction in the trastuzumab clinical trials experience. Journal of Clinical Oncology. 2002;20(5):1215-21.

3. Pai V, Nahata M. Cardiotoxicity of chemotherapeutic agents: incidence, treatment and prevention. Drug Saf. 2000;22:263 - 302. PubMed.

4. Dolci A, Dominici R, Cardinale D, Sandri MT, Panteghini M. Biochemical Markers for Prediction of Chemotherapy-Induced Cardiotoxicity: Systematic Review of the Literature and Recommendations for Use. Am J Clin Pathol. 2008;130(5):688-95. PubMed. 
5. Singal P, Iliskovic N. Doxorubicin-induced cardiomyopathy. The New England Journal of Medicine. 1998;339(13):900-5.

6. Carvalho F, Burgeiro A, Garcia R, Moreno A, Carvalho R, Oliveira P. Doxorubicin-Induced Cardiotoxicity: From Bioenergetic Failure and Cell Death to Cardiomyopathy. Medicinal Research Reviens. 2014;34(1):106-35.

7. Dell RB, Holleran S, Ramakrishnan R. Sample size determination. Ilar Journal. 2002;43(4):207.

8. Nousiainen T, Jantunen E, Vanninen E, Hartikainen $\mathrm{J}$. Early decline in left ventricular ejection fraction predicts doxorubicin cardiotoxicity in lymphoma patients. $\mathrm{Br}$ J Cancer. 0000;86(11):1697-700. PubMed.

9. Ehler D, Carney DK, Dempsey AL, Rigling R, Kraft C, Witt SA, et al. Guidelines for cardiac sonographer education: recommendations of the American Society ofEchocardiography Sonographer Training and Education Committee. Journal of the American Society of Echocardiography. 2001;14(1):77-84.

10. Kremer L, Van Dalen E, Offringa M, Voute P. Frequency and risk factors of anthracycline-induced clinical heart failure in children: a systematic review. Annals of Oncology. 2002;13(4):503-12.

11. Khattry N, Varma S, Malhotra P, Grover A, Sharma S. Doxorubicin induced cardiotoxicity in adult Indian patients on chemotherapy. ASCO Meeting Abstracts. 2005;23(16_suppl):2123-.

12. Limat S, Daguindau E, Cahn JY, Nerich V, Brion A, Perrin S, et al. Incidence and risk-factors of $\mathrm{CHOP} / \mathrm{R}$ CHOP-related cardiotoxicity in patients with aggressive non-Hodgkin's lymphoma. Journal of Clinical Pharmacy and Therapentics. 2014;39(2):168-74.

13. Kremer L, Van der Pal H, Offringa M, Van Dalen E, Voute P. Frequency and risk factors of subclinical cardio- toxicity after anthracycline therapy in children: a systematic review. Annals of Oncology. 2002;13(6):819-29.

14. Shaikh A, Saleem A, Mohsin S, Alam M, Ahmed M. Anthracycline-induced cardiotoxicity: prospective cohort study from Pakistan. BMJ Open. 2013;3:e003663.

15. Elalouani C, Benhmidoun MA, Rida H, AitRaiss M, Derhem N, Elomrani A, et al. [Short and medium term cardiotoxicity of anthracyclins: a prospective study]. Ann Cardiol Angeiol (Paris). 2012;61(4):257-66.

16. Pio M, Adoubi K, Adoh A, Didi-Kouko C, Anzouan-Kacou JB, Tegnan A, et al. [Early detection of anthracyclines cardiotoxicity by tissue Doppler echocardiography about 45 cases at Abidjan institute of cardiology]. Ann Cardiol Angeiol (Paris). 2013;62(1):28-33.

17. Shiroya-Wandabwa M, Yuko-Jowi C, Nduati R, Githang'a J, Walmalwa D. Risk factors for cardiac dysfunction in children on treatment for cancer at Kenyatta National Hospital, Nairobi. East African medical journal. 2009;86(12):52-7.

18. Kremer L, van der Pal H, Offringa M, van Dalen E, Voute P. Frequency and risk factors of subclinical cardiotoxicity after anthracycline therapy in children: a systematic review (Structured abstract). Annals of Oncology. 2002;13:81 -29. PubMed.

19. Schwartz C, Hobbie W, Truesdell S, Constine L, Clark E. Corrected QT interval prolongation in anthracycline-treated survivors of childhood cancer. J Clin Oncol. 1993;11(10):1906-10.

20. Yeh E, Tong A, Lenihan D, et-al. Cardiovascular Complications of Cancer Therapy: Diagnosis, Pathogenesis, and Management. Circulation. 2004;109:3122-31. PubMed.

21. Larsen R, Jakacki R, Vetter V, Meadows A, Silber J, Barber G. Electrocardiographic changes and arrhythmias after cancer therapy in children and young adults. The American Journal of Cardiology. 1992;70(1):73-7. 